\title{
CHEVALLeY BASES FOR LIE MODULES (')
}

BY

DAVID A. SMITH

1. Introduction. In Theorem 1 of [3], Chevalley established the existence of a basis for a complex semi-simple Lie algebra $\ell_{C}$ with certain special properties. One property is of integral multiplication table, making it possible to define an analogous Lie algebra $\mathfrak{l}$ over an arbitrary field $K$. Another (related) property is the fact that the linear transformations $(n !)^{-1}\left(\operatorname{ad} e_{\alpha}\right)^{n}$ (where $e_{\alpha}$ is a root vector in the basis and $n$ a positive integer) have integral matrices, making it possible to associate the automorphisms of $\mathfrak{l}$ with those of $\mathfrak{R}_{C}$ (see e.g. [11], [12]). We will observe first that this latter property characterizes Chevalley bases (in an appropriate sense). Thought of as a property of the adjoint representation, it has a natural generalization to other representations, and will be used to define Chevalley bases for an $\mathfrak{R}_{C^{-}}$ module. $\$ 3$ and 4 will be devoted to proving the existence of such a basis for a finite-dimensional irreducible $\mathfrak{l}_{C}$-module $\mathfrak{M}_{C}$.

Since the original preparation of this paper, $R$. Ree has published a paper [13] which contains a proof of the existence of Chevalley bases for modules. His proof depends on the Cartan classification of the algebras $\mathfrak{R}_{C}$, and he states, "a direct proof of [ the existence] is desirable." While Theorem 2 of the present paper is not quite as strong as Ree's Theorem (1.6), the proof presented here is direct and entirely different from Ree's. Furthermore, essentially the same consequences for algebras, modules, and groups over arbitrary fields can be obtained except that Theorem 2 below is useless in passing to a field of characteristic 2 or 3 for some purposes (see \$5). It should also be noted that Ree's proof depends on Chevalley's basis theorem, and the present proof does not.

A Chevalley basis for $\mathfrak{M}_{C}$ will be used to associate with $\mathfrak{M}_{C}$ (or with the corresponding representation $R$ ) an $\mathfrak{R}$-module $\mathfrak{M}$ and a linear group $G^{R}$ analogous to the Chevalley group $G^{\prime}$ of automorphisms of $\mathbb{R}$. A general fixed point theorem for the groups $G^{R}$, analogous to Theorem 1 of [11], is proved in \$6. Application of this theorem to the case of a simple Lie algebra of type $F_{4}$ shows that every automorphism of an exceptional central simple Jordan algebra over an arbitrary field of characteristic $\neq 2,3$ has at least a threedimensional fixed point space. Other applications of the theorem yield known results about fixed points of rotations in odd-dimensional spaces and fixed points of automorphisms of Cayley algebras.

Received by the editors March 6, 1964.

( ${ }^{1}$ Part of a dissertation presented for the degree of Doctor of Philosophy in Yale University. The author wishes to thank Professor Nathan Jacobson, who directed this research. 
2. A characterization of Chevalley bases. Let $\mathbb{R}$ be a finite-dimensional

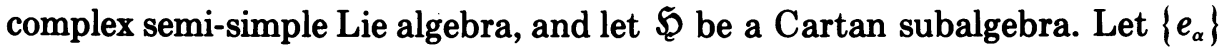
be a complete set of root vectors for the (nonzero) roots $\alpha$ of $\mathfrak{l}$ with respect to $\mathfrak{S}$. We write

$$
\left[e_{\alpha} e_{\beta}\right]=N_{\alpha, \beta} e_{\alpha+\beta}
$$

if $\alpha+\beta$ is a root. Set $N_{\alpha, \beta}=0$ if $\alpha+\beta$ is not a root (and not zero), and then (1) holds for all pairs $\alpha, \beta$ if $\alpha \neq-\beta$. We will denote the $\alpha$-string of roots through $\beta$ by

$$
\beta-r_{\alpha \beta} \alpha, \cdots, \beta, \beta+\alpha, \cdots, \beta+q_{\alpha \beta} \alpha .
$$

Let $\mathfrak{R}_{\alpha}$ denote the root space of $\alpha$, and let $h_{\alpha}$ be the unique element of $\left[\mathfrak{R}_{\alpha}, \mathfrak{R}_{-\alpha}\right]$ such that $\alpha\left(h_{\alpha}\right)=2$.

A set $\left\{e_{\alpha}\right\}$ of root vectors can always be replaced by a set of scalar multiples of themselves so that the new set satisfies

$$
\left[e_{\alpha} e_{-\alpha}\right]=h_{\alpha} \text {. }
$$

Assuming this done, we have [3, pp. 21-23]

$$
N_{\alpha, \beta} N_{-\alpha,-\beta}=\left(r_{\alpha \beta}+1\right)^{2} \text {. }
$$

(The reason for a difference in sign between (3) and the formula given in [3] is that Chevalley takes the adjoint mappings to act on the left instead of on the right.)

The essence of the Chevalley basis theorem [3, p.24] is that the $e_{\alpha}$ may again be replaced by scalar multiples of themselves, preserving (2) and so that $N_{\alpha, \beta}=N_{-\alpha,-\beta}$. Hence $\left[e_{\alpha} e_{\beta}\right]= \pm\left(r_{\alpha \beta}+1\right) e_{\alpha+\beta}$. The root vectors can then be taken as part of a basis having an integral multiplication table, and having the further property that the matrices of the linear transformations $(n !)^{-1}\left(\operatorname{ad} e_{\alpha}\right)^{n}$, for all roots $\alpha$ and positive integers $n$, have only integer entries. This property, in the presence of (2), characterizes Chevalley bases, and in fact can be stated in a somewhat weaker form. Henceforth let $\Pi$ denote a fixed fundamental system of roots.

THEOREM 1. Let $\mathfrak{R}$ have a basis consisting of a basis for $\mathfrak{S}$ and a complete set $\left\{e_{\beta}\right\}$ of root vectors such that

(a) $\left[e_{\beta} e_{-\beta}\right]=h_{\beta}$ for all roots $\beta$;

(b) the matrices of the linear transformations $(n !)^{-1}\left(\operatorname{ad} e_{\alpha}\right)^{n}$ have integer entries for $\pm \alpha \in \Pi, n=1,2, \cdots$.

Then $N_{\alpha, \beta}=N_{-\alpha,-\beta}$ for all pairs of roots $\alpha, \beta$, and hence the $e_{\beta}$ 's are root vectors in a Chevalley basis.

Proof. Let $\alpha \in \Pi$, and let $\beta$ be a root such that $\alpha+\beta$ is not a root, i.e., $q_{\alpha \beta}=0$ and $r_{\alpha \beta}$ is some integer $r$. Then for $k=1,2, \cdots, r$, we have 


$$
\begin{aligned}
e_{\beta}\left(\operatorname{ad} e_{-\alpha}\right)^{k} & =N_{\beta,-\alpha} N_{\beta-\alpha,-\alpha}^{\prime} \cdots N_{\beta-(k-1) \alpha,-\alpha} e_{\beta-k \alpha}, \\
e_{-\beta}\left(\operatorname{ad} e_{\alpha}\right)^{k} & =N_{-\beta, \alpha} N_{-\beta+\alpha, \alpha} \cdots N_{-\beta+(k-1) \alpha, \alpha} e_{-\beta+k \alpha}
\end{aligned}
$$

The N's are all integers ((b) for $n=1$ ) and (a) implies that (3) holds. We prove by induction that

$$
N_{-\beta+k \alpha, \alpha}=N_{\beta-k \alpha,-\alpha}= \pm(k+1), \quad k=0,1, \cdots, r-1 .
$$

For $k=0$ we have $N_{-\beta, \alpha} N_{\beta,-\alpha}=\left(r_{-\alpha \beta}+1\right)^{2}=1$, and hence $N_{-\beta, \alpha}=N_{\beta,-\alpha}$ $= \pm 1$. Assuming (6) for $k-1,(4)$ and (5) give

$$
\begin{aligned}
& e_{\beta}\left(\operatorname{ad} e_{-\alpha}\right)^{k+1}= \pm k ! N_{\beta-k \alpha,-\alpha} e_{\beta-(k+1) \alpha}, \\
& e_{-\beta}\left(\operatorname{ad} e_{\alpha}\right)^{k+1}= \pm k ! N_{-\beta+k \alpha, \alpha} e_{-\beta+(k+1) \alpha} .
\end{aligned}
$$

Thus (b) implies that $k+1$ divides both $N_{\beta-k \alpha,-\alpha}$ and $N_{-\beta+k \alpha, \alpha}$. Since

$$
N_{\beta-k \alpha,-\alpha} N_{-\beta+k \alpha, \alpha}=\left(r_{-\alpha, \beta-k \alpha}+1\right)^{2}=(k+1)^{2},
$$

(6) follows for $k$, which completes the induction step.

Now consider an arbitrary root $\beta$ with $\alpha$-string

$$
\beta-r \alpha, \cdots, \beta, \cdots, \beta+q \alpha .
$$

Let $\beta^{\prime}=-\beta+r \alpha$. Then $\beta^{\prime}+\alpha$ is not a root, and

$$
N_{\beta, \alpha}=N_{-\beta^{\prime}+r \alpha, \alpha}= \pm(r+1)=N_{\beta^{\prime}-r \alpha,-\alpha}=N_{-\beta,-\alpha} .
$$

Thus we have $N_{\beta, \alpha}=N_{-\beta,-\alpha}$ for $\beta$ an arbitrary root and $\alpha \in \Pi$. Since $\beta$ can be replaced by $-\beta$, this also holds for $-\alpha \in \Pi$. Consider the roots ordered lexicographically by $\Pi$. Our result thus far is the initial step for proving the desired conclusion for an arbitrary $\alpha>0$ (and hence for all $\alpha$ ) by induction on the ordering.

Thus let $\gamma>0$ be arbitrary, but not in $\Pi$, and assume $N_{\beta, \alpha}=N_{-\beta,-\alpha}$ for all $\beta$ and all $\alpha<\gamma$. Write $\gamma=\alpha+\delta$, where $\alpha$ and $\delta$ are positive roots $<\gamma$. For an arbitrary root $\beta$, the Jacobi identity for $e_{\beta}, e_{\alpha}, e_{\delta}$ yields

$$
N_{\beta, \gamma} N_{\delta, \alpha}=N_{\beta, \delta} N_{\beta+\delta, \alpha}+N_{\alpha, \beta} N_{\alpha+\beta, \delta}
$$

Similarly, for $e_{-\beta}, e_{-\alpha}, e_{-\delta}$, we get

$$
N_{-\beta,-\gamma} N_{-\delta,-\alpha}=N_{-\beta,-\delta} N_{-\beta-\delta,-\alpha}+N_{-\alpha,-\beta} N_{-\alpha-\beta,-\delta}
$$

In all the factors of (10) except the first we have a subscript of $-\alpha$ or $-\delta$, and the induction hypothesis gives

$$
N_{-\beta,-\gamma} N_{\delta, \alpha}=N_{\beta, \delta} N_{\beta+\delta, \alpha}+N_{\alpha, \beta} N_{\alpha+\beta, \delta}
$$

Since $\alpha+\delta$ is a root, namely $\gamma, N_{\delta, \alpha} \neq 0$, and (9) and (11) imply $N_{\beta, \gamma}$ $=N_{-\beta,-\gamma}$, which completes the induction step, and the theorem.

3. Module bases. In this section $\mathfrak{R}$ will denote a finite-dimensional split 
semi-simple Lie algebra over an arbitrary field $K$ of characteristic 0 . Let $\left(A_{i j}\right)$ be the Cartan matrix of $\mathcal{R}$ and $e_{i}, f_{i}, h_{i}(1 \leqq i \leqq l)$ a set of canonical generators $[8$, p.126] for $\mathfrak{R}$. Let $\mathfrak{M}$ be a finite-dimensional irreducible $\mathfrak{R}$-module with associated representation $R$.

Definition. A Chevalley basis for $\mathfrak{M}$ is any basis of weight vectors with respect to which the linear transformations

$$
(m !)^{-1}\left(e_{i}^{R}\right)^{m},(m !)^{-1}\left(f_{i}^{R}\right)^{m}, \quad 1 \leqq i \leqq l, \quad m=1,2, \cdots,
$$

have matrices with integer entries.

REMARK. In [13], Ree calls a basis for $\mathfrak{M}$ regular if it satisfies the condition above for all the root vectors of a Chevalley basis of $\mathfrak{R}$, not just the canonical generators. It is for this reason that $[13,(1.6)]$ is stronger than the following theorem.

Theorem 2. Every finite-dimensional irreducible $\mathfrak{P}_{\text {-module }} \mathfrak{M}$ has a Chevalley basis.

The purpose of this section and the next will be the proof of this theorem. The main ideas in the proof will be sketched first, and the computational details, isolated as Lemma 1 below, will be deferred until \$4.

We begin by constructing $\mathfrak{M}$ as in Jacobson [8, Chapter VII]. Let $\mathfrak{F}$ be the free Lie algebra on $3 l$ generators $e_{i}, f_{i}, h_{i}(1 \leqq i \leqq l)$, and let $\widetilde{\mathfrak{l}}=\mathfrak{F} / \mathfrak{F}$, where $\mathcal{Y}$ is the ideal generated by the elements

$$
\begin{aligned}
& {\left[h_{i} h_{j}\right],} \\
& {\left[e_{i} f_{j}\right]-\delta_{i j} h_{i},} \\
& {\left[e_{i} h_{j}\right]-A_{j i} e_{i},} \\
& {\left[f_{i} h_{j}\right]+A_{j i} f_{i} .}
\end{aligned}
$$

Let $\mathfrak{Q}$ be the span of the $h_{i}$ 's in $\mathfrak{F}$, and let $\alpha_{i}$ be the linear function on $\mathfrak{Q}$ such that $\alpha_{i}\left(h_{j}\right)=A_{j i}, i, j=1,2, \ldots, l$. Let $\Lambda$ be a dominant integral linear function on $\mathfrak{Q}$, i.e., $\Lambda\left(h_{i}\right)$ is a non-negative integer for $i=1,2, \cdots, l$. Let $\mathfrak{X}$ be the free algebra (associative with identity) on $l$ generators $x_{1}, x_{2}, \ldots, x_{l}$. $\mathfrak{X}$ becomes a module for $\mathfrak{F}$ and for $\widetilde{\mathfrak{l}}$ by the definitions

$$
\begin{aligned}
\left(x_{i_{1}} \cdots x_{i_{r}}\right) h_{i} & =\left(\Lambda-\alpha_{i_{1}}-\cdots-\alpha_{i_{r}}\right)\left(h_{i}\right) x_{i_{1}} \cdots x_{i_{r}}, \\
\left(x_{i_{1}} \cdots x_{i_{r}}\right) f_{i} & =x_{i_{1}} \cdots x_{i_{r}} x_{i}, \\
1 e_{i} & =0 \\
\left(x_{i_{1}} \cdots x_{i_{r}}\right) e_{i} & =\left(\left(x_{i_{1}} \cdots x_{i_{r-1}}\right) e_{i}\right) x_{i_{r}} \\
& \quad-\delta_{i, i_{r}}\left(\Lambda-\alpha_{i_{1}}-\cdots-\alpha_{i_{r}-1}\right)\left(h_{i}\right) x_{i_{1}} \cdots x_{i_{r-1}} .
\end{aligned}
$$

Here we understand that $x_{i_{1}} \cdots x_{i_{r}}=1$ if $r=0$. The algebra $\mathfrak{l}=\tilde{\mathfrak{l}} / \mathfrak{J}^{\prime}$, 
where $\mathcal{Y}^{\prime}$ is the intersection of the kernels of all finite-dimensional irreducible representations of $\widetilde{\mathfrak{R}}$. The same names for the generators $e_{i}, f_{i}, h_{i}$ have been used throughout because the space spanned by them is mapped isomorphically in passing from $\mathfrak{F}$ to $\mathfrak{P}$. $\mathfrak{X}$ has a unique maximal submodule $\mathfrak{B} ; \mathfrak{X} / \mathfrak{B}$ is irreducible and finite-dimensional, hence is an $\mathbb{R}$-module $\mathfrak{M}$. This turns out to be the unique finite-dimensional irreducible $\mathbb{R}$-module with highest weight $\Lambda$.

A basis for $\mathfrak{M}$ may be found by selecting a linearly independent set of cosets modulo $\mathfrak{B}$ of monomials $x_{i_{1}} \ldots x_{i_{r}}$ in $\mathfrak{X}$. Modification of such a basis by suitable scalar multiplications will yield a Chevalley basis. Specifically, write an arbitrary monomial as $x_{i_{1}}^{k_{1}} \ldots x_{i_{r}}^{k_{r}}$, where the highest possible exponents are displayed. We call $\left(k_{1} ! \ldots k_{r} !\right)^{-1} x_{i_{1}}^{k_{1}} \ldots x_{i_{r}}^{k_{r}} a$ "modified monomial."

LEMMA 1. The image of a modified monomial under each of the mappings (12) is an integral linear combination of modified monomials.

Now specialize the base field $K$ to the rational field $Q$. Modified monomials in $\mathfrak{X}$ are weight vectors (by (14)). For each weight $\lambda$, let $\mathfrak{V}_{\lambda}$ be the additive group generated by the cosets of the modified monomials belonging to $\lambda$. $\mathfrak{V}_{\lambda}$ is finitely generated, and hence has a basis. Such a basis is also a basis for the $Q$-space spanned by $\mathfrak{V}_{\lambda}$, which is the weight space $\mathfrak{M}_{\lambda}$ of $\mathfrak{M}$. We take as basis for $\mathfrak{M}$ the union of these bases for weight spaces. Since every basis element for $\mathfrak{M}$ is an integral linear combination of cosets of modified monomials, and every such coset is an integral linear combination of basis elements, the mappings (12) have integral matrices with respect to the basis chosen for $\mathfrak{M}$.

The result is extended to an arbitrary base field $K$ of characteristic 0 by identifying the prime field of $K$ with $Q$ and constructing $\mathfrak{R}_{K}$ and $\mathfrak{M}_{K} \cdot \mathfrak{R}_{K}$ is the split semi-simple Lie algebra over $K$ with Cartan matrix $\left(A_{i j}\right)$, and since $\mathfrak{M}$ is absolutely irreducible $[8, \mathrm{p} .223], \mathfrak{M}_{K}$ is the finite-dimensional irreducible $\mathfrak{R}_{K}$-module with highest weight $\Lambda$. The Chevalley basis for $\mathfrak{M}$ is also one for $\mathfrak{M}_{K}$. The proof of Lemma 1 is all that remains to complete the proof of Theorem 2.

REMARK. The combination of Theorems 1 and 2 does not yield an alternative proof of the Chevalley basis theorem, because of the necessity of hypothesis (a) in Theorem 1, which does not seem to have a module analogue. The combination does provide a somewhat weaker theorem which is adequate for defining the groups of Chevalley - but only on the basis of Chevalley's own results on the generation of these groups [3, Lemmas III.4 and IV.3].

4. Proof of Lemma 1. Half of the lemma is quite easy to prove. Let

$$
\left(k_{1} ! \cdots k_{r} !\right)^{-1} x_{i_{1}}^{k_{1}} \cdots x_{i_{r}}^{k_{r}}
$$


be a modified monomial. We have

$$
\left(k_{1} ! \cdots k_{r} !\right)^{-1} x_{i_{1}}^{k_{1}} \ldots x_{i_{r}}^{k_{r}}(m !)^{-1}\left(f_{i}^{R}\right)^{m}=\left(k_{1} ! \cdots k_{r} ! m !\right)^{-1} x_{i_{1}}^{k_{1}} \ldots x_{i r}^{k_{r}} x_{i}^{m},
$$

which gives a coefficient of 1 if $i \neq i_{r}$; otherwise, we have

$$
\begin{aligned}
\left(k_{1} ! \cdots k_{r} ! m !\right)^{-1} x_{i_{1}}^{k_{1}} \cdots & x_{i_{r-1}}^{k_{r}-1} x_{i_{r}}^{k_{r}+m} \\
& =\left(\begin{array}{c}
k_{r}+m \\
k_{r}
\end{array}\right)\left(k_{1} ! \cdots k_{r-1} !\left(k_{r}+m\right) !\right)^{-1} x_{i_{1}}^{k_{1}} \cdots x_{i_{r-1}}^{k_{r}-1} x_{i_{r}+m}^{k_{r}} .
\end{aligned}
$$

Thus the statement is correct for the $f_{i}^{\prime}$ 's.

In the sequel we will have to frequently write linear combinations

$$
k_{1} \alpha_{i_{1}}+\cdots+k_{r} \alpha_{i_{r}}
$$

of roots, sometimes with complicated subscripts. We denote such a linear combination by $k \alpha_{i}[1, r]$. If each $k_{i}=1$, we write $\alpha_{i}[1, r]$.

It is useful to replace (16) with the explicit form for the operation of $e_{i}$ on a monomial, which is:

$$
\left(x_{i_{1}} \cdots x_{i_{r}}\right) e_{i}=-\sum_{j=1}^{r} \delta_{i_{j} i}\left(\Lambda-\alpha_{i}[1, j-1]\right)\left(h_{i}\right) x_{i_{1}} \cdots \hat{x}_{i_{j}} \cdots x_{i_{r}},
$$

where denotes deletion of the argument. This follows immediately from (16) by induction on $r$. Thus the image of a monomial under $e_{i}$ is a linear combination of monomials each of which is obtained from the original by deletion of exactly one $x_{i}$.

Next we note the significance of having more than one $x_{i}$ appearing together in a monomial. On a monomial of the form $x_{i_{1}} \ldots x_{i}^{k} \ldots x_{i_{r}}$, operation by $e_{i}$ produces (inter alia) $k$ terms all involving the monomial

$$
x_{i_{1}} \cdots x_{i}^{k-1} \ldots x_{i_{r}}
$$

which can thus be combined. If $x_{i j}$ is the first $x_{i}$ in the group being considered, the sum of the coefficients of these terms is:

$$
\begin{aligned}
-k\left(\Lambda-\alpha_{i}[1, j-1]\right) & \left(h_{i}\right)-\sum_{m=0}^{k-1} m \alpha_{i}\left(h_{i}\right) \\
& =-k\left\{\left(\Lambda-\alpha_{i}[1, j-1]\right)\left(h_{i}\right)-k+1\right\} .
\end{aligned}
$$

The next task is to compute explicitly the effect of $\left(e_{i}^{R}\right)^{m}$ on an arbitrary monomial. To do this, we need to display all the powers of $x_{j}^{\prime}$ 's appearing in the monomial, singling out the $x_{i}$ 's. The reader is asked to visualize a monomial $X$ with sequence of subscripts

$$
i_{1}, \cdots, i_{r_{1}} ; i ; i_{r_{1}+1}, \cdots, i_{r_{2}} ; i ; i_{r_{2}+1}, \cdots, i_{r_{n}} ; i ; i_{r_{n}+1}, \cdots, i_{r_{n+1}} \text {, }
$$

and corresponding sequence of exponents 
(20) $k_{1}, \cdots, k_{r_{1}} ; N_{1} ; k_{r_{1}+1}, \cdots, k_{r_{2}} ; N_{2} ; k_{r_{2}+1}, \cdots, k_{r_{n}} ; N_{n} ; k_{r_{n}+1}, \cdots, k_{r_{n+1}}$,

where we may have $r_{1}=0$ and/or $r_{n+1}=r_{n}$, but adjacent subscripts must be distinct. Now $X e_{i}^{m}$ will be a linear combination of monomials, a typical one of which can be obtained from $X$ by replacing $x_{i}^{N_{j}}$ by $x_{i}^{N_{j-m_{j}}}(1 \leqq j \leqq n)$, where $m_{1}+m_{2}+\cdots+m_{n}=m$. In other words, a typical term of $X e_{i}^{m}$ is associated with an "ordered partition" $m=m_{1}+\cdots+m_{n}$ of the integer $m$, subject to $0 \leqq m_{j} \leqq N_{j}$ for all $j$, and in fact there is a term of $X e_{i}^{m}$ for each such ordered partition (possibly with zero coefficient, or course).

We need to consider the $\alpha_{i}$-strings of weights through each of the weights $\lambda_{j}=\Lambda-k \alpha_{i}\left[1, r_{j}\right]-\left(\sum_{1}^{j} N_{v}\right) \alpha_{i}, \quad 1 \leqq j \leqq n$. The $j$ th such string will start with $\lambda_{j}-p_{j} \alpha_{i}$ and end with $\lambda_{j}+q_{j} \alpha_{i}$. Then we have [8, Theorem 4.1]:

$$
\left(\Lambda-k \alpha_{i}\left[1, r_{j}\right]\right)\left(h_{i}\right)=p_{j}-q_{j}+2 \sum_{1}^{j} N_{v}
$$

For convenience, we introduce the following abbreviations for expressions which recur frequently:

$$
\begin{aligned}
T_{j} & =p_{j}-q_{j}+N_{j}, \\
M_{j} & =m_{1}+m_{2}+\cdots+m_{j} \quad\left(M_{0}=0, M_{n}=m\right), \\
A_{j} & =T_{j}+M_{j}, \\
B_{j} & =T_{j}+M_{j-1}, \\
C_{j} & =A_{j}+M_{j-1}=B_{j}+M_{j},
\end{aligned}
$$

where in each case $j=1,2, \cdots, n$.

We prove by induction on $m$ that the term of $X e_{i}^{m}$ corresponding to the ordered partition $m=m_{1}+\cdots+m_{n}$ has the coefficient

$$
(-1)^{m} m ! \prod_{j=1}^{n}\left[\coprod_{t=0}^{m_{j}-1}\left(N_{j}-t\right)\left(\begin{array}{c}
A_{j} \\
B_{j}
\end{array}\right)\right] .
$$

For $m=1$, we have some $m_{i}=1$, all others $=0$. The assertion is that we get a coefficient of $-N_{j}\left(T_{j}+1\right)$. By (18), we get a coefficient of

$$
\begin{aligned}
-N_{j} & \left\{\left(\Lambda-k \alpha_{i}\left[1, r_{j}\right]-\sum_{1}^{j-1} N_{v} \alpha_{i}\right)\left(h_{i}\right)-N_{j}+1\right\} \\
& =-N_{j}\left(p_{j}-q_{j}+2 \sum_{1}^{j} N_{v}-2 \sum_{1}^{j-1} N_{v}-N_{j}+1\right) \\
& =-N_{j}\left(p_{j}-q_{j}+N_{j}+1\right)=-N_{j}\left(T_{j}+1\right)
\end{aligned}
$$

as required. 
Now assume the assertion is true for $m-1$. There are exactly $n$ terms of $X e_{i}^{m-1}$ which contribute to the term of $X e_{i}^{m}$ under consideration, namely, those corresponding to the ordered partitions $m-1=m_{1}+\cdots+m_{s-1}$ $+\left(m_{s}-1\right)+m_{s+1}+\cdots+m_{n}$. By the induction hypothesis, the $s$ th one of these has coefficient

$$
(-1)^{m-1}(m-1) ! \prod_{t=0}^{m_{1}-1}\left(N_{1}-t\right) \ldots \prod_{t=0}^{m_{s}-2}\left(N_{s}-t\right) \ldots \prod_{t=0}^{m_{n}-1}\left(N_{n}-t\right)
$$

$$
\cdot\left(\begin{array}{c}
A_{1} \\
B_{1}
\end{array}\right) \cdots\left(\begin{array}{c}
A_{s-1} \\
B_{s-1}
\end{array}\right)\left(\begin{array}{c}
A_{s}-1 \\
B_{s}
\end{array}\right)\left(\begin{array}{c}
A_{s+1}-1 \\
B_{s+1}-1
\end{array}\right) \cdots\left(\begin{array}{c}
A_{n}-1 \\
B_{n}-1
\end{array}\right) .
$$

The contribution of the term with coefficient (23) to $X e_{i}^{m}$ is, by (18) and (21), another factor of:

$$
\begin{aligned}
-\left(N_{s}\right. & \left.-m_{s}+1\right)\left\{\left(\Lambda-k \alpha_{i}\left[1, r_{s}\right]-\sum_{1}^{s-1}\left(N_{v}-m_{v}\right) \alpha_{i}\right)\left(h_{i}\right)-N_{s}+m_{s}\right\} \\
& =-\left(N_{s}-m_{s}+1\right)\left(p_{s}-q_{s}+2 \sum_{1}^{s} N_{v}-2 \sum_{1}^{s-1}\left(N_{v}-m_{v}\right)-N_{s}+m_{s}\right) \\
& =-\left(N_{s}-m_{s}+1\right)\left(T_{s}+2 M_{s-1}+m_{s}\right) \\
& =-\left(N_{s}-m_{s}+1\right) C_{s} .
\end{aligned}
$$

The coefficient we seek is obtained by summing the product of (23) and (24) for $s=1,2, \cdots, n$. Clearly the following factors are common to every term of the sum:

$$
(-1)^{m}(m-1) ! \prod_{j=1}^{n} \prod_{t=0}^{m_{j}-1}\left(N_{j}-t\right) .
$$

The rest of the sum, which must be factored into the remaining factors of (22), is:

$$
\begin{aligned}
\sum_{s=1}^{n} & C_{s}\left(\begin{array}{c}
A_{1} \\
B_{1}
\end{array}\right) \cdots\left(\begin{array}{c}
A_{s-1} \\
B_{s-1}
\end{array}\right)\left(\begin{array}{c}
A_{s}-1 \\
B_{s}
\end{array}\right)\left(\begin{array}{c}
A_{s+1}-1 \\
B_{s+1}-1
\end{array}\right) \cdots\left(\begin{array}{c}
A_{n}-1 \\
B_{n}-1
\end{array}\right) \\
& =\sum_{s=1}^{n} C_{s} \prod_{1}^{s-1} A_{w} ! \prod_{s}^{n}\left(A_{w}-1\right) !\left\{\prod_{1}^{s} B_{w} ! \prod_{s+1}^{n}\left(B_{w}-1\right) !\left(m_{s}-1\right) ! \prod_{w \neq s} m_{w} !\right\}^{-1} \\
& =\prod_{1}^{n}\left(B_{w} ! m_{w} !\right)^{-1} \sum_{s=1}^{n} m_{s}\left(\prod_{s+1}^{n} B_{w}\right) C_{s} \prod_{1}^{s-1} A_{w} ! \prod_{s}^{n}\left(A_{w}-1\right) ! \\
& =\prod_{1}^{n}\left(A_{w}-1\right) !\left(B_{w} ! m_{w} !\right)^{-1} \sum_{s=1}^{n} m_{s} A_{1} \cdots A_{s-1} C_{s} B_{s+1} \cdots B_{n} .
\end{aligned}
$$

We now proceed to factor the sum remaining in (26). We note first that $C_{1}=T_{1}+m_{1}=T_{1}+M_{1}=A_{1}$, and one can verify from the definitions that 


$$
m_{w} C_{w}=M_{w} A_{w}-M_{w-1} B_{w}, \quad w=1,2, \cdots, n .
$$

Then the sum in (26) becomes

$$
\begin{gathered}
\sum_{s=1}^{n} M_{s} A_{1} A_{2} \cdots A_{s} B_{s+1} \cdots B_{n}-\sum_{w=0}^{n-1} M_{w} A_{1} \cdots A_{w} B_{w+1} \cdots B_{n} \\
=M_{n} A_{1} A_{2} \cdots A_{n}=m A_{1} A_{2} \cdots A_{n} .
\end{gathered}
$$

Hence (26) becomes

$$
\left(m \prod_{1}^{n} A_{w} !\right) /\left(\prod_{1}^{n} B_{w} ! m_{w} !\right)=m \prod_{1}^{n}\left(\begin{array}{c}
A_{w} \\
B_{w}
\end{array}\right) .
$$

The product of (28) and (25) gives (22) as required to complete the induction step.

The modified monomial corresponding to the monomial $X$ defined by (19) and (20) is $\left(k_{1} ! \cdots k_{r_{n+1}} ! N_{1} ! \cdots N_{n} !\right)^{-1} X$. The monomial in the term of $X e_{i}^{m}$ corresponding to $m=m_{1}+\cdots+m_{n}$ gets replaced by

$$
\left(k_{1} ! \ldots k_{r_{n+1}} !\left(N_{1}-m_{1}\right) ! \ldots\left(N_{n}-m_{n}\right) !\right)^{-1}
$$

times itself. Thus, the coefficient of this term must be an integral multiple of $m ! \prod_{j=1}^{n} \prod_{t=0}^{n_{j-1}}\left(N_{i}-t\right)$, which is precisely what (22) asserts it is.

This completes the proof of Lemma 1 and hence also of Theorem 2.

5. Analogues of the Chevalley groups. In this section the notation of $[11, \S 2]$ will be used. In particular, $\ell_{C}$ denotes a complex semi-simple Lie algebra with a fixed Chevalley basis $\left\{h_{i}, e_{\alpha}\right\}$, and $\mathbb{R}$ the corresponding Lie algebra of Chevalley over an arbitrary field $K$ of characteristic $\neq 2,3$. (This restriction on characteristics will be assumed henceforth without further mention.)

If $\alpha, \beta$ and $\alpha+\beta$ are roots, then

$$
\left[e_{\alpha} e_{\beta}\right]=N_{\alpha, \beta} e_{\alpha+\beta},
$$

where $N_{\alpha, \beta}$ is an integer, and $1 \leqq\left|N_{\alpha, \beta}\right| \leqq 4$. Thus each $e_{\alpha}$ can be written as a rational multiple of a product of the form $\left[\ldots\left[\left[e_{i_{1}} e_{i_{2}}\right] e_{i_{3}}\right] \cdots e_{i_{r}}\right]$ or of the form $\left[\cdots\left[\left[f_{i_{1}} f_{i_{2}}\right] f_{i_{3}}\right] \cdots f_{i_{r}}\right]$, where the multiplier (in lowest terms) has only powers of 2 and 3 in the denominator. It will be convenient in the sequel to refer to the subring $B$ of the rational field $Q$ consisting of all rationals having only 2 and 3 as prime factors of their denominators (when in the lowest terms). If $p$ is a prime $>3$, then $B / p B$ is a field of $p$ elements and will be identified with the prime field $K_{0}$ of any field $K$ of characteristic $p$.

Let $\Lambda$ be a dominant integral linear function on $\mathfrak{S}_{c}$ with respect to the basis $h_{1}, h_{2}, \ldots, h_{l}$. Let $\mathfrak{M}_{C}$ be the corresponding finite-dimensional irreducible $\mathfrak{R}_{C}$-module, and $R$ the associated representation. Let $y_{1}, \cdots, y_{n}$ be a Chevalley basis for $\mathfrak{M}_{\mathcal{C}}$, in the sense defined above, which will henceforth be 
fixed. When convenient, linear transformations in $\mathfrak{M}_{C}$ will be identified with matrices relative to this basis.

We now describe a process for associating with the $\mathfrak{R}_{C}$-module $\mathfrak{M}_{C}$ an $\mathfrak{R}$-module $\mathfrak{M}$. The matrices $e_{i}^{R}, f_{i}^{R}, h_{i}^{R}$ have integer entries, and by the remarks above, $e_{\alpha}^{R}$ is a matrix with entries in $B$ for every root vector $e_{\alpha}$ in the Chevalley basis. Reduce the entries of each of the matrices $h_{i}^{R}, e_{\alpha}^{R}$ modulo the characteristic of $K$. The result is a set of matrices over the prime field $K_{0}$ of $K$. Considering the elements $\left\{h_{i}, e_{\alpha}\right\}$ as the Chevalley basis of $\mathfrak{R}$, and continuing to denote the matrices over $K_{0}$ by $h_{i}^{R}, e_{\alpha}^{R}$, we have a linear mapping $R: h_{i} \rightarrow h_{i}^{R}, e_{\alpha} \rightarrow e_{\alpha}^{R}$ of $\mathcal{R}$ into the space of $n$-by- $n$ matrices over $K$.

It is easy to verify that $R$ is a matrix representation of $\mathcal{L}$, and as such has an associated module $\mathfrak{M}$, considering the matrices in $\mathfrak{R}^{k}$ as linear transformations of the $K$-space $\mathfrak{M}$ with respect to a fixed basis. We have $\operatorname{dim}_{K} \mathfrak{M}=n$ $=\operatorname{dim}_{C} \mathfrak{M}_{C}$, and it is convenient to identify the Chevalley basis $y_{1}, \ldots, y_{n}$ of $\mathfrak{M}_{C}$ with the fixed basis in $\mathfrak{M}$.

We recall the definition of the Chevalley group $G^{\prime}$. Let

$$
x(t ; \alpha)=\exp \left(t \operatorname{ad} e_{\alpha}\right),
$$

for $t \in C$ and $\alpha$ a root of $\mathscr{R}_{C}$. (The notation $x_{\alpha}(t)$ is used in [11].) $x(t ; \alpha)$ is an automorphism of $\mathfrak{R}_{C}$ and has a matrix (relative to the Chevalley basis) with entries which are polynomials in $t$ with integer coefficients. Replace the complex parameter $t$ by an indeterminate, and then specialize the indeterminate to an arbitrary element $t \in K$. The result, also denoted $x(t ; \alpha)$, is the matrix relative to the Chevalley basis of an automorphism of $\mathbb{R}$. The group $G^{\prime}$ is the group generated by the automorphisms $x(t ; \alpha)$ for all roots $\alpha$ of $\mathfrak{R}_{C}$ and all $t \in K$. Actually, $G^{\prime}$ is generated by the $x(t ; \alpha)$ for $t \in K$ and $\pm \alpha \in \Pi$, a fixed fundamental system of roots $[3$, p. 48$]$.

We now define groups similar to $G^{\prime}$ by the use of Theorem 2 . Let $x(t ; \alpha, R)$ $=\exp t e_{\alpha}^{R}, \pm \alpha \in \Pi, t \in C$, where $\Pi=\left\{\alpha_{1}, \alpha_{2}, \cdots, \alpha_{l}\right\}$. Relative to the Chevalley basis of $\mathfrak{M}_{C}$, each $x(t ; \alpha, R)$ has a matrix of determinant 1 with entries which are polynomials in $t$ with integer coefficients. As above, replace $t$ by an indeterminate, and then specialize the indeterminate to an arbitrary element $t \in K$. The result is a nonsingular matrix, again denoted $x(t ; \alpha, R)$, or equivalently, a nonsingular linear transformation of $\mathfrak{M}$. Let $G^{R}$ denote the linear group generated by the $x(t ; \alpha, R)$ for all $t \in K, \pm \alpha \in \Pi$.

Theorem 3. If $K$ is algebraically closed, then $G^{R}$ is an irreducible algebraic group.

Proof. We first observe that the mapping $t \rightarrow x(t ; \alpha, R)$ for a fixed $\alpha$ is a homomorphism of $(K,+)$ into $G^{R}$. For any complex numbers $t, t^{\prime}$ we have $x\left(t+t^{\prime} ; \alpha, R\right)=x(t ; \alpha, R) x\left(t^{\prime} ; \alpha, R\right)$. Replacing the complex parameters by indeterminates, we get a matrix identity in which the entries are polynomials 
with integer coefficients. Specialization to $t, t^{\prime} \in K$ gives the fact that $t \rightarrow x(t ; \alpha, R)$ is a homomorphism.

Let $X(\alpha, R)$ be the group of all $x(t ; \alpha, R), t \in K$. Then $X(\alpha, R)$ is the image of $(K,+)$ under the rational representation $t \rightarrow x(t ; \alpha, R)$. Hence $X(\alpha, R)$ is an irreducible algebraic group $[2, \mathrm{pp} .115,112]$. Since $G^{R}$ is generated by irreducible algebraic groups, it is also irreducible algebraic $[2$, p. 123].

6. The fixed point theorem. For the time being, let $\mathfrak{l}$ denote a finitedimensional semi-simple Lie algebra over an algebraically closed field of characteristic 0 . Let $\mathfrak{M}$ be a finite-dimensional irreducible $\mathfrak{R}$-module with associated representation $R$, and let $k$ denote the multiplicity of 0 as a weight of $\mathfrak{M}$. For each $a \in \mathfrak{R}$, let $\mathfrak{Z}_{a}=\left\{z \in \mathfrak{M} \mid z a^{R}=0\right\}$.

Lemma $2 . \operatorname{dim} \mathfrak{Z}_{a} \geqq k$ for each $a \in \mathfrak{R}$.

Proof. First let $a$ be a regular element. Then the Fitting null component $\mathfrak{W}_{a}$ of ad $a$ is a Cartan subalgebra containing $a\left[8\right.$, p.59]. Let $\mathfrak{M}_{0}$ denote the 0 weight space of $\mathfrak{M}$ relative to $\mathfrak{S}_{a}$. Then $\mathfrak{M}_{0} \subseteq \mathfrak{Z}_{a}$, and $k=\operatorname{dim} \mathfrak{M}_{0}$ $\leqq \operatorname{dim} \boldsymbol{Z}_{a}$.

Now let $a_{1}, a_{2}, \cdots, a_{n}$ be a basis for $\mathfrak{Q}$, and let $\xi_{1}, \xi_{2}, \cdots, \xi_{n}$ be algebraically independent indeterminates. Let $\mathrm{P}=\Phi\left(\xi_{1}, \ldots, \xi_{n}\right)$, the field of rational expressions in the $\xi_{i}$, and consider $\mathfrak{R}_{\mathrm{P}}$ and $\mathfrak{M}_{\mathrm{P}}$ obtained by extension of the base field. The generic element $x=\sum \xi_{i} a_{i}$ of $\mathfrak{R}$ is a regular element of $\mathfrak{R}_{\mathrm{p}}$, hence also of $\mathfrak{R}_{\Omega}$, where $\Omega$ is the algebraic closure of $P$ [8, pp.66-61]. If $Z_{x}$ denotes the null space of $x^{R}$ in $\mathfrak{M}_{\mathrm{a}}$ (where $R$ is used to denote the extension of itself), then $\operatorname{dim}_{\Omega} Z_{x} \geqq k$, by the first part of the proof. Hence rank $x^{R}$ $=\operatorname{dim} \mathfrak{M}_{\Omega}-\operatorname{dim} \mathfrak{Z}_{x} \leqq \operatorname{dim} \mathfrak{M}_{\Omega}-k=\operatorname{dim}_{\Phi} \mathfrak{M}-k$. An arbitrary element $a \in \mathfrak{R}$, say $a=\sum t_{i} a_{i}$, is the image of $x$ under the specialization $\xi_{i} \rightarrow t_{i}$. Thus rank $a^{R} \leqq \operatorname{rank} x^{R} \leqq \operatorname{dim} \mathfrak{M}-k$, so $\operatorname{dim} \mathfrak{Z}_{a} \geqq k$.

Let $e_{\alpha}$ denote a root vector of $\mathfrak{R}$ for each (nonzero) root $\alpha$ relative to a Car$\tan$ subalgebra $\mathfrak{S}$. Let $\xi_{1}, \cdots, \xi_{r}$ be indeterminates, and let $P$ now denote the field of formal power series over $\Phi$ in the $\xi_{i}$ 's (i.e., the quotient field of the algebra of formal power series). Consider $\mathfrak{R}_{\mathrm{p}}$ and the nonsingular linear transformation $\tau(\xi)=\left(\exp \xi_{1} e_{\alpha_{1}}^{R}\right) \ldots\left(\exp \xi_{r} e_{\alpha_{r}}^{R}\right)$ of $\mathfrak{M}_{\mathrm{p}}$, where the $\alpha_{i}$ are arbitrary roots. Let $\mathfrak{N}$ be the fixed point space of $\tau(\xi)$ in $\mathfrak{M}_{\mathrm{p}}$.

LEMMA $3 . \operatorname{dim} \mathfrak{N} \geqq k$.

Proof. Form the free algebra $\mathfrak{X}$ and the free Lie algebra $\mathfrak{F}$ on $r$ generators $\eta_{1}, \cdots, \eta_{r} . \mathfrak{X}$ may be extended to the algebra $\overline{\mathfrak{X}}=\Phi\left[\left[\eta_{1}, \cdots, \eta_{r}\right]\right]$ of formal power series in the $\eta_{i}$ 's, and $\mathfrak{F}$ may be extended to the Lie subalgebra $\bar{F}$ of power series, each whose homogeneous terms is a Lie element of $\mathfrak{X}$, i.e., an element of $\mathfrak{\xi}$. Let $\tau(\eta)=\left(\exp \eta_{1}\right) \cdots\left(\exp \eta_{r}\right) \in \overline{\mathfrak{X}}$. The Campbell-Hausdorff 
formula [8, p. 172] gives $\tau(\eta)=\exp \zeta$, where $\zeta \in \overline{\mathfrak{F}}$. The canonical mapping $\overline{\mathfrak{F}} \rightarrow \Phi\left[\left[\xi_{1} e_{\alpha_{1}}^{R}, \cdots, \xi_{r} e_{\alpha_{r}}^{R}\right]\right]$ maps $\tau(\eta) \rightarrow \tau(\xi)$ and $\exp \zeta \rightarrow \exp A$, where $A$ is a Lie element, i.e., a power series in the elements of the Lie algebra generated by $\xi_{1} e_{\alpha_{1}}^{R}, \cdots, \xi_{r} e_{\alpha_{r}}^{R}$. Since ad $e_{\alpha_{i}}$ is nilpotent in $\mathbb{R}$, ad $\xi_{i} e_{\alpha_{i}}^{R}$ is nilpotent in $\mathbb{R}_{\mathrm{p} \text {. }}^{R}$ Hence one may apply the Specht-Wever Theorem (see e.g. [8, pp. 169, 173]) to show that there are only a finite number of nonzero terms in the power series $A$. Hence $A \in \mathfrak{R}_{\mathrm{p}}^{K}$, which means $A=a^{K}$ for some $a \in \mathfrak{R}_{\mathrm{p}}$, and $\tau(\xi)$ $=\exp a^{K}$. Now let $z \in \mathfrak{Z}_{a}=\left\{z \in \mathfrak{M}_{\mathrm{P}} \mid z a^{K}=0\right\}$. Then $z \tau(\zeta)=z$, and hence $\mathfrak{Z}_{a} \subseteq \mathfrak{N}$. If $\Omega$ denotes the algebraic closure of $\mathrm{P}$, we have, by Lemma 2, $\operatorname{dim}_{\mathrm{P}} \mathfrak{Z}_{a}=\operatorname{dim}_{\Omega}\left(\mathfrak{Z}_{a}\right)_{\Omega} \geqq k$, and therefore $\operatorname{dim}_{\mathrm{P}} N \geqq k$.

We now resume the notation of $\S 5$. $\mathfrak{R}_{C}$ denotes a complex semi-simple Lie algebra, and $\mathbb{R}$ the associated Lie algebra over an arbitrary field $K$ of characteristic $\neq 2,3$. Let $\mathfrak{M}_{C}$ be an irreducible $\mathfrak{R}_{C}$-module and $\mathfrak{M}$ the associated $\mathbb{R}$-module. Let $R$ be the corresponding representation in either case, and let $G^{K}$ be the group defined in $\$ 5$. For each $\tau \in G^{K}$, let $\mathfrak{F}(\tau)$ denote the fixed point space of $\tau$ in $M$.

TheOREM 4. For each $\tau \in G^{k}, \operatorname{dim} \mathfrak{F}(\tau) \geqq k$, the multiplicity of 0 as a weight of $\mathfrak{R}_{C}$ in $\mathfrak{M}_{C}$.

Proof. Write $\tau=x\left(t_{1} ; \alpha_{1}, R\right) \cdots x\left(t_{r} ; \alpha_{r}, R\right)$. As in Lemma 3 , let $\xi_{1}, \cdots, \xi_{r}$ be indeterminates, $\mathrm{P}$ the field of formal power series over $C$ in the $\xi_{i}$ and $\tau(\xi)=\left(\exp \xi_{1} e_{\alpha_{1}}^{R}\right) \cdots\left(\exp \xi_{r} e_{\alpha_{r}}^{R}\right)$. The element $\tau$ of $G^{R}$ is obtained by observing that relative to a Chevalley basis for $\mathfrak{M}_{C}$ (which is also a basis for $\left.\left(\mathfrak{N}_{C}\right)_{\mathrm{P}}\right)$ the matrix $\tau(\xi)$ has entries which are polynomials in the $\xi_{i}$ 's with coefficients in the ring $B$ defined in $\$ 5$, and then specializing $\xi_{i} \rightarrow t_{i}$ in $K$. By Lemma 3 , the space $\mathfrak{N}$ of $\tau(\xi)$-fixed points in $\left(\mathfrak{M}_{C}\right)_{\mathrm{P}}$ has dimension $\geqq k$. Thus rank $(\tau(\zeta)-I) \leqq \operatorname{dim}\left(\mathfrak{M}_{\mathcal{C}}\right)_{\mathrm{p}}-k=\operatorname{dim}_{K} \mathfrak{M}-k$. Hence $\operatorname{rank}(\tau-I)$ $=\operatorname{dim} \mathfrak{M}-\operatorname{dim} \mathfrak{F}(\tau) \leqq \operatorname{dim} \mathfrak{M}-k$, and $\operatorname{dim} \mathfrak{F}(\tau) \geqq k$.

COROLlary. If 0 is a weight of $R$ (in $\mathfrak{M}_{\mathcal{C}}$ ), then every element of $G^{K}$ has a (nonzero) fixed point.

The following criterion is useful in determining when the fixed point theorem is applicable.

Theorem (Freudenthal [4]). Zero is a weight of $\mathfrak{M}_{C}$ if and only if the highest weight is a sum of fundamental roots.

If we take our usual basis $h_{1}, h_{2}, \cdots, h_{l}$ for $\mathfrak{S}_{C}$, each dominant integral linear function $\Lambda$ on $\mathfrak{S}_{C}$ is a sum of the basic highest weights $\lambda_{1}, \lambda_{2}, \cdots, \lambda_{l}$ defined by $\lambda_{i}\left(h_{j}\right)=\delta_{i j}$. Thus one can determine whether 0 is a weight of $\mathfrak{M}_{C}$ for a given $\Lambda$ by examining the coefficients of the $\lambda_{i}$ written as linear combinations of the $\alpha_{i}$ (see e.g. [5, p. 318]). 
7. Automorphisms of exceptional simple Jordan algebras. Application of Theorem 4 to the 26-dimensional irreducible module for the Lie algebra of type $F_{4}$ will be seen to give information about the fixed points of automorphisms of exceptional simple Jordan algebras. We assume familiarity with the notation, computational formulas, and constructions in Seligman [10, $\$ \$ 1-2]$. Unexplained notations are defined there.

Let $\Im$ be the split exceptional simple Jordan algebra over an arbitrary field $K$ (which will continue to mean characteristic $\neq 2,3$ ). Let $\mathbb{R}$ be the simple Lie algebra over $K$ of classical type $F_{4}$. Then $\mathbb{R}$ can be represented as the derivation algebra of $\mathfrak{Y}$. We take as a fundamental system of roots for $\mathbb{R}$ the roots $\alpha_{1}, \alpha_{2}, \alpha_{3}, \alpha_{4}$ defined on p. 293 of [10]. Let $\mathbb{R}_{\alpha}$ denote the root space of $\alpha$ in $\mathfrak{R}$. Then the elements $h_{i}^{\prime}$ of $\left[\mathfrak{R}_{\alpha_{i}}, \mathfrak{R}_{-\alpha_{i}}\right]$ such that $\alpha_{i}\left(h_{i}^{\prime}\right)=2(i=1,2,3$, 4) are given by:

$$
\begin{aligned}
& h_{1}^{\prime}=h_{2}-h_{3}, \\
& h_{2}^{\prime}=h_{1}-h_{2}, \\
& h_{3}^{\prime}=2 h_{1}, \\
& h_{4}^{\prime}=-h_{1}+h_{2}+h_{3}+h_{4},
\end{aligned}
$$

as may be verified by $[10,(14),(15),(16),(18)]$. A set of root vectors $e_{i}, f_{i}(1 \leqq i \leqq 4)$ for the $\pm \alpha_{i}$ such that $\left\{e_{i}, f_{i}, h_{i}^{\prime} \mid 1 \leqq i \leqq 4\right\}$ is a canonical set of generators is given by:

$$
\begin{array}{ll}
e_{1}=2\left(0,0,0, E_{32}-E_{67}\right), & f_{1}=2\left(0,0,0, E_{76}-E_{23}\right), \\
e_{2}=2\left(0,0,0, E_{16}-E_{25}\right), & f_{2}=2\left(0,0,0, E_{52}-E_{61}\right), \\
e_{3}=2\left(u_{1}, 0,0,0\right), & f_{3}=\left(u_{5}, 0,0,0\right), \\
e_{4}=2\left(0, u_{5}, 0,0\right), & f_{4}=\left(0, u_{1}, 0,0\right) .
\end{array}
$$

Again, the fact that $\left[e_{i} f_{i}\right]=h_{i}^{\prime}$ for each $i$ can be verified by $[10,(14)]$. For calculating the effect of the $e_{i}$ and $f_{i}$ as derivations of $\mathcal{Y}$, one also needs to observe that $\left(E_{32}-E_{67}\right)^{\psi}=\left(E_{32}-E_{67}\right)^{\phi}=E_{32}-E_{67},\left(E_{16}-E_{25}\right)^{\psi}$ $=\left(E_{16}-E_{25}\right)^{\varphi}=4\left(E_{83}-E_{74}\right)$ and similar expressions for the effects of $\varphi$ and $\psi$ on the skew transformations appearing in the definitions of $f_{1}$ and $f_{2}$. This follows from [10, pp. 293-294].

We select the following fixed basis for $\Im: 1, w_{1}=\operatorname{diag}\{1,-1,0\}$, $w_{2}=\operatorname{diag}\{0,1,-1\}, 2^{m(4)} u_{1}(1,2), \cdots, 2^{m(11)} u_{8}(1,2), 2^{m(12)} u_{1}(1,3), \ldots$, $2^{m(19)} u_{8}(1,3), 2^{m(20)} u_{1}(2,3), \cdots, 2^{m(27)} u_{8}(2,3)$, where the exponents $m(j)$ take the following values for $j=4,5, \cdots, 27: 0,-1,-1,4,-1,0,0,-5 ;-1$, $-2,-2,3,0,1,1,-4 ; 3,2,2,-1,-4,-3,-3,0$.

Let $(i, j)$ denote a 27 -by-27 matrix unit with 1 in the $(i, j)$-position. Then relative to this basis for $\mathcal{Y}$, the $e_{i}$ and $f_{i}$ have the following matrices $[10 ;(20)]$ : 


$$
\begin{aligned}
e_{1}= & (6,5)-(9,10)+(14,13)-(17,18)+(22,21)-(25,26), \\
f_{1}= & (10,9)-(5,6)+(18,17)-(13,14)+(26,25)-(21,22), \\
e_{2}= & (4,9)-(5,8)+(19,14)-(18,15)+(27,22)-(26,23), \\
f_{2}= & (8,5)-(9,4)+(15,18)-(14,19)+(23,26)-(22,27), \\
e_{3}= & 2(2,4)-(3,4)-(8,2)-(13,26)+(14,25)-(15,20) \\
& +(16,27)-(21,18)+(22,17)-(23,12)+(24,19), \\
f_{3}= & 2(2,8)-(3,8)-(4,2)+(12,23)-(17,22)+(18,21) \\
& -(19,24)+(20,15)-(25,14)+(26,13)-(27,16), \\
e_{4}= & (2,16)+(3,16)+(4,27)+(9,22)-(10,21)+(11,24) \\
& -(12,2)-(12,3)-(20,7)-(23,8)+(25,6)-(26,5), \\
f_{4}= & (2,12)+(3,12)+(5,26)-(6,25)+(7,20)+(8,23) \\
& -(16,2)-(16,3)+(21,10)-(22,9)-(24,11)-(27,4) .
\end{aligned}
$$

From these expressions we may compute directly:

$$
\begin{aligned}
& e_{1}^{2}=f_{1}^{2}=e_{2}^{2}=f_{2}^{2}=0, \\
& e_{3}^{2}=-2(8,4), f_{3}^{2}=-2(4,8), \\
& e_{4}^{2}=-2(12,16), f_{4}^{2}=-2(16,12), \\
& e_{3}^{3}=f_{3}^{3}=e_{4}^{3}=f_{4}^{3}=0 .
\end{aligned}
$$

THEOREM 5. Every automorphism of an exceptional central simple Jordan algebra $\Im$ over an arbitrary field of characteristic $\neq 2,3$ has at least $a$ 3-dimensional space of fixed points.

Proof. Let $\Omega$ be the algebraic closure of the base field $K$. Then $\Im_{\Omega}$ is a necessarily split exceptional simple Jordan algebra over $\Omega$, and since fixed point spaces are preserved under field extension, it suffices to prove the theorem in the algebraically closed case. Thus we may assume $\Im$ itself is split.

Let $\Im_{C}$ denote the exceptional simple Jordan algebra over the complex field, and $\mathfrak{R}_{C}$ its derivation algebra. The restriction of $\mathfrak{R}_{C}$ to the space $\Im_{C}^{\prime}$ of elements of trace 0 gives a 26-dimensional irreducible representation of $\mathfrak{l}_{C}$, so we set $\mathfrak{M}_{C}=\Im_{C}^{\prime}$. If we apply the previous contents of this section to $\Im_{C}$, we see that the basis for $\mathfrak{M}_{C}$ obtained by dropping the first basis element for $\Im_{c}$ is in fact a Chevalley basis relative to the selected canonical generators for $\mathfrak{R}_{C}$. The full basis for $\Im_{C}$ has a multiplication table with coefficients in the ring $B$. Thus when we pass to our arbitrary field $K$, we get not only $\mathbb{R}$ and $\mathfrak{M}$, but also the split exceptional Jordan algebra $\Im$. Furthermore, 
$\mathfrak{M}=\mathfrak{Y}^{\prime}$, the space of elements of trace 0 in $\mathcal{Y}$, and $\mathfrak{R}$ is the Lie algebra of derivations of $\Im$.

The zero weight space in $\mathfrak{M}_{C}$ is spanned by $w_{1}$ and $w_{2}$. Thus Theorem 4 asserts that every element of the group $G^{k}$ generated by the mappings $x(t ; \alpha, R)(t \in K, \pm \alpha \in \Pi)$ of $\mathfrak{M}$ has at least a 2-dimensional fixed point space.

We note that $e_{\alpha}^{R}$ denotes a nilpotent linear transformation of $\mathfrak{M}_{C}$ (or $\left.\mathfrak{M}\right)$; it may be extended uniquely to a derivation of $\Im_{C}$ (or $\Im$ ) by setting $1 e_{\alpha}^{R}=0$. Similarly, $x(t ; \alpha, R)(t \in C)$ will also denote the extension of itself to $\Im_{C}$ defined by $1 \cdot x(t ; \alpha, R)=1$. Clearly, this is the exponential of the extended $t e_{\alpha}^{R}$, a nilpotent derivation, and hence is an automorphism of $\Im_{c}$. Passing from $C$ to $K$ in the usual way gives automorphisms $x(t ; \alpha, R)$ of $\Im$. In this way $G^{R}$ can be considered as a subgroup of $\mathscr{U}(\mathcal{I})$, the automorphism group of $\Im$. since each element of $G^{R}$ has at least a 3-dimensional fixed point space in $\Im$, the proof will be complete when we show that $G^{R}=\mathscr{H}(\mathcal{Y})$.

If $A \in \mathscr{A}(\Im)$, then $I_{A}: X \rightarrow A^{-1} X A$ for $X \in \mathcal{R}$ defines an automorphism of $\mathfrak{R}$, and $A \rightarrow I_{A}$ is a monomorphism of $\mathscr{U}(\mathcal{Y})$ into $\mathscr{A}(\mathfrak{R})$. The image of $x(t ; \alpha, R)$ is $x(t ; \alpha), \pm \alpha \in \Pi, t \in K$ (by $[9$, p. 454, (2)], since in this case $\left(e_{\alpha}^{R}\right)^{3}=0$, and $\left.x(t ; \alpha, R)=\exp t e_{\alpha}^{R}\right)$. The Chevalley group $G^{\prime}$ is generated by the $x(t ; \alpha)$ for $\pm \alpha \in \Pi, t \in K$, and in this case is the full automorphism group of $\mathfrak{R}[12,(4.8)]$, so $A \rightarrow I_{A}$ maps $G^{R}$ onto $\mathscr{U}(\mathfrak{R})$ and therefore

$$
G^{R}=\mathfrak{U}(\Im) \cong \mathscr{U}(\mathfrak{R}) \text {. }
$$

The next task is to show that the lower bound in Theorem 5 is actually achieved. For this purpose, familiarity with the notation and terminology of Jacobson [7] (particularly the proof of the triality principle in \$2) is assumed. Theorem 6 will be proved by exhibiting an automorphism that achieves the lower bound, in a manner analogous to the proof of Theorem 2 of $[11]$.

THEOREM 6. The minimal dimension of fixed point spaces of automorphisms of a split exceptional simple Jordan algebra $\Im$ over a field $K$ of characteristic $\neq 2,3$, or 5 is 3 .

Proof. Continuing the notation of [10] with respect to $\mathcal{Y}$ and the split Cayley algebra $\mathfrak{C}$ over $K$, we select the following elements $b_{1}, \cdots, b_{8}$ of $\mathfrak{E}$ :

$$
\begin{gathered}
b_{1}=u_{4}+u_{8}, \quad b_{2}=u_{4}-\frac{1}{2} u_{8} \\
b_{2 i+1}=2 u_{i}-\frac{1}{2} u_{i+4}, \quad i=1,2,3 \\
b_{2 i+2}=-u_{i}-\frac{1}{2} u_{i+4}, \quad i=1,2,3 .
\end{gathered}
$$


The multiplication table for $[10$, p. 287] may be used to calculate the matrices of the symmetries $S_{1}, S_{b_{i}}$, with respect to the identity and to the $b_{i}$, and we find that

$$
A_{1} \equiv \prod_{i=1}^{8} S_{1} S_{b_{i}}=\operatorname{diag}\left\{-\frac{1}{2},-\frac{1}{2},-\frac{1}{2},-\frac{1}{2},-2,-2,-2,-2\right\} .
$$

The orthogonal transformations $A_{2}, A_{3}$ related to $A_{1}$ by the triality principle $[7$, p. $78,(13)]$ are given by

$$
A_{2}=\beta \prod_{i=1}^{8} n\left(b_{i}\right)^{-1} b_{i L}, \quad A_{3}=\beta^{-1} \prod_{i=1}^{8} b_{i R}
$$

where $\beta^{2}=\prod_{i=1}^{\dot{\delta}} n\left(b_{i}\right)\left[7\right.$, pp. 75, 79]. We have $n\left(b_{i}\right)=2$ for $i$ odd and -1 for $i$ even, so $\prod_{i=1}^{8} n\left(b_{i}\right)=16$. We take $\beta=-4$. The left and right multiplications by the $b_{i}$ may also be computed from the multiplication table, and we find:

$$
\begin{aligned}
& A_{2}=\operatorname{diag}\left\{2,2,2,2, \frac{1}{2}, \frac{1}{2}, \frac{1}{2}, \frac{1}{2}\right\}, \\
& A_{3}=\operatorname{diag}\left\{-1,-1,-1,-\frac{1}{4},-1,-1,-1,-4\right\} .
\end{aligned}
$$

$A_{1}, A_{2}$, and $A_{3}$ define an automorphism of $\Im$ [7, p. 87, Proof of Theorem 6] whose fixed point space is the 3-dimensional space spanned by the diagonal idempotents. This, together with Theorem 5 , completes the proof.

Other simple cases in which the fixed point theorem can be applied are the irreducible representations of minimal degree for $\mathfrak{R}_{C}$ of types $B_{l}$ and $G_{2}$. In the first case one gets the known result that every rotation in an odddimensional space over an arbitrary field (here of characteristic $\neq 2,3$ ) has a nonzero fixed point $[1$, p. 131]. In the second case, the result is that every automorphism of a Cayley algebra has a fixed point in the 7-dimensional subspace of elements of trace 0 . This result is also known, and in fact is a special case of the previous one, since automorphisms of Cayley algebras act in the trace 0 space as rotations with respect to the norm form [6]. In each case, the details of applying the fixed point theorem are similar to the $F_{4}$ case worked out above. Application of Theorem 4 to adjoint representations also yields a part of the result of [11, Theorem 1].

\section{REFERENCES}

1. E. Artin, Geometric algebra, Interscience, New York, 1957.

2. C. Chevalley, Theorie des groupes de Lie. II, Actualités Sci. Indust. No. 1152, Hermann, Paris, 1951. 
3. Sur certains groupes simples, Tôhoku Math. J. (2) 7(1955), 14-66.

4. H. Freudenthal, The existence of a vector of weight 0 in irreducible Lie groups without centre, Proc. Amer. Math. Soc. 7(1956), 175-176.

5. _ Lie groups, Lithographed notes, Yale Univ., New Haven, Conn., 1961.

6. N. Jacobson, Composition algebras and their automorphisms, Rend. Circ. Mat. Palermo (2) 7(1958), 55-80.

7. , Some groups of transformations defined by Jordan algebras. II, J. Reine Angew. Math. $204(1960), 74-98$.

8. , Lie algebras, Interscience, New York, 1962.

9. G. B. Seligman, On automorphisms of Lie algebras of classical type. II, Trans. Amer. Math. Soc. 94(1960), 452-482.

10. __ On automorphisms of Lie algebras of classical type. III, Trans. Amer. Math. Soc. 97(1960), 286-316.

11. D. A. Smith, On fixed points of automorphisms of classical Lie algebras, Pacific J. Math. 14(1964), 1079-1089.

12. R. Steinberg, Automorphisms of classical Lie algebras, Pacific J. Math. 11(1961), 11191129 .

13. R. Ree, Construction of certain semi-simple groups, Canad. J. Math. 16(1964), 490-508.

Duke University,

Durham, North Carolina 